\title{
Assessment Strategies for Learner-Centered Software
}

\author{
Chris Quintana, Eric Fretz, Joseph Krajcik, and Elliot Soloway \\ Center for Highly Interactive Computing in Education, University of Michigan \\ 1101 Beal Ave., Ann Arbor, MI 48109 \\ Tel: 734-763-6988, Fax: 734-763-1260 \\ Email: quintana@umich.edu
}

\begin{abstract}
Learners are novices in some work domain that they are trying to work in and learn. Learner-centered design (LCD) is a design approach aimed at developing software to support learners via scaffolding as they try to work in and learn the given work domain. Here, we focus on methods to assess the effectiveness of scaffolding strategies by presenting an initial assessment framework for observing the local "effects with" individual scaffolded features and the global "effects of" the overall software. We describe dimensions by which we can assess scaffolding. We also describe strategies for understanding the "cognitive residue" of the software on the learner to define a more concrete guide for assessing learner-centered scaffolding.
\end{abstract}

Keywords: learner-centered design, scaffolding, assessment, design process

\section{Introduction: Background and Motivation}

Learner-centered design (LCD) is an evolving design approach addressing the needs of learners-a specific audience trying to work in and understand a work domain in which they are novices (Soloway et al. 1996, Soloway et al. 1994). The central tenet of learner-centered design involves the design of scaffolding into software to make complex tasks accessible. Scaffolding allows learners to engage in unknown work activities that would normally be out of their reach, and in turn, begin developing an understanding of the work (Soloway et al. 1994).

As we develop learner-centered software (e.g., Quintana et al. 1999), a major challenge that we face is how to assess scaffolding so as to understand the cognitive effects on learners. Traditional controlled assessment techniques can be difficult to use for LCD. Additionally, there are questions as to whether controlled laboratory testing results will generalize to the "real-world" classroom context of use (Chi 1997). Some software assessment methods show how learners use software and what they understand about a work domain through surveys, videotaped software usage, interviews, etc. Other methods analyze qualitative observational data in a more quantitative manner to effectively assess software without controlled studies (e.g., Chi 1997). These are all effective assessment techniques. However, we want to construct a more systematic assessment guide that incorporates many of these ideas to describe how different scaffolding strategies are effective in addressing learner needs.

\section{Issues in Assessing Learner-Centered Software}

Assessment of learner-centered software should involve methods for evaluating individual scaffolded features in the software as a learner uses them over time. Assessment should also evaluate the overall effectiveness of the software to see how well a learner understands the work domain after software use. Thus, we must evaluate the different cognitive effects resulting from technology use, including (Salomon et al. 1991):

- "Effects with" technology, i.e., the changes in performance that students display as they use the software. By evaluating these "local effects" of technology, we analyze how learners use different scaffolded features.

- "Effects of" technology, i.e., the changes in the learner's understanding of the work domain (or the "cognitive residue") after they have used the software. By evaluating these "global effects" of technology, we analyze the effectiveness of the software as a whole.

\section{Assessing the "Effects With" Technology}

Assigning a binary "good" or "bad" rating to each scaffolded feature is difficult. Instead, we would like to assess software along different dimensions for finer shades of assessment information. Our approach involves taking each scaffolded feature in a software package and evaluating the individual features along each of the following dimensions (as applicable) as the learner uses the software over an extensive period of time: 
- Initial Accessibility: Involves a "yes/no" answer to show whether learners can access or use a scaffolded feature. If a scaffolded feature is not usable or accessible, then the feature should be redesigned.

- Efficiency: Measures how fast learners use a particular scaffolded feature and how their performance changes over time to see if the software might be interfering with the completion of their work tasks.

- Accuracy: Measures whether a scaffolded feature supports the correct and appropriate "doing" of a work task and whether the learner's accuracy improves over time to see if additional work support is needed.

- Progression: Measures how learners progress through their work tasks while using a given scaffolded feature to see if they work in a linear step-by-step manner (i.e., a novice workstyle) or in a more opportunistic, iterative style over time. An opportunistic, iterative workstyle is more indicative of expert work (Schoenfeld 1987) and would show that the learner is developing a model of the work domain.

- Reflectiveness: Measures the amount of reflecting that a learner performs while using a scaffolded feature. This describes whether the learner's cognitive focus is on their work tasks and how their reflection varies over time to see if additional support for working in a "mindful" manner is necessary (Salomon et al 1991).

\section{Assessing the "Effects Of" Technology}

Aside from local scaffolding assessment information, we also need to gather global information to assess what learners understand about the target work domain after using the tool. Now we use more traditional assessment techniques to observe the residual effects of software use by observing what learners know about the target work domain before and after software use. We determine baseline assessments of the learner's work knowledge by having learners complete initial tasks from the work domain, whether on the computer, through interviews, or using a paper and pencil prototype. We then observe transfer tasks after software use to understand the learner's work knowledge. This again involves asking the learner questions about the work domain and completing work activities. We then compare performance on the initial tasks with the transfer task performance.

\section{Future Directions and Concluding Remarks}

We are still researching the assessment framework, but are now in a position to incorporate the framework in our development process to assess our software. In the future, we need to explore the following issues. First, we want to list the specific information described by the different "effects with" dimensions to see how we can subject these observations to more quantitative assessment methods (Chi 1997). Second, we would like to better define how the "effects of" and "effects with" categories are connected to show how different individual scaffolding strategies contribute to the overall understanding of the target work domain being exhibited by the learner.

\section{References}

Chi, M. (1997) Quantifying Qualitative Analyses of Verbal Data: A Practical Guide. Journal of the Learning Sciences, 6(3), 271-315.

Quintana, C., Eng, J., Carra, A., Wu, H., Soloway, E. (1999) Symphony: A Case Study in Extending LearnerCentered Design Through Process Space Analysis. Proceedings of CHI '99 (Pittsburgh, May) ACM Press.

Salomon, G., Perkins, D. N., Globerson, T. (1991) Partners in Cognition: Extending Human Intelligence with Intelligent Technologies. Educational Researcher, April, pp. 2-9.

Schoenfeld, A. H. (1987) What's All The Fuss About Metacognition? In A.H. Schoenfeld (Ed.) Cognitive Science and Mathematics Education, Lawrence Erlbaum Associates, Hillsdale NJ, 1987.

Soloway, E, Jackson, S.L, Klein, J., Quintana, C., Reed, J., Spitulnik, J., Stratford, S.J., Studer, S., Eng, J., and Scala, N. (1996) Learning Theory in Practice: Case Studies of Learner-Centered Design. Proceedings of CHI '96 (Vancouver, Canada, April) ACM Press.

Soloway, E., Guzdial, M., and Hay, K.E. (1994). Learner-centered design: The challenge for HCI in the 21st century. Interactions. 1(2): p. 36-48.

\section{Acknowledgements}

This work is supported by the National Science Foundation. 